\title{
Selected Abstracts from the 3rd National Conference \\ of the Society of Fetal Medicine, 1-3 August, 2014, Kochi, Kerala (India)
}

Published online: 7 May 2015

(C) Society of Fetal Medicine 2015

\section{“Star Gazing Fetus": Sign of Iniencephaly on Antenatal Ultrasonography}

\section{Glossy B. Sabharwal}

Department of Radiodiagnosis, Jeewan Mala Hospital, New Delhi

e-mail: docglossy@gmail.com

Introduction Iniencephaly is a rare neural tube defect characterized by extreme retroflexion of the head with absence of the neck due to spinal deformities. The defective 'inion' (posterior-most part of occipital bone) fuses with the back resulting in the absence of neck and fixed retroflexion of head. Incidence in India is estimated to be one in 65,000 deliveries. Overall reported incidence is higher and ranges from 0.1 to 10 in 10,000 deliveries. Majority of cases are females (90\%).

Report of Case The authors report a 33-year-old multigravida woman who visited for routine obstetric checkup at 15 weeks of gestation. She had no prior antenatal checkup or folic acid supplementation. She had been suffering from hypothyroid for four years and was on thyroxine supplementation (50 mcg once daily). The marriage was nonconsanguineous. There was no history of teratogen exposure.

Patient's obstetric history was G6P1A4L1. In the first pregnancy, she delivered a full-term healthy male child weighing $1.75 \mathrm{~kg}$. The second pregnancy required medical termination of pregnancy because of intrauterine fetal demise. The third, fourth and fifth pregnancies had ended in spontaneous abortions at six, and seven weeks gestation, respectively. No investigations were done in the previous cases as patient's family was not willing.

Laboratory tests including complete blood picture, blood sugar, urinalysis, HIV, HBsAg, and VDRL were

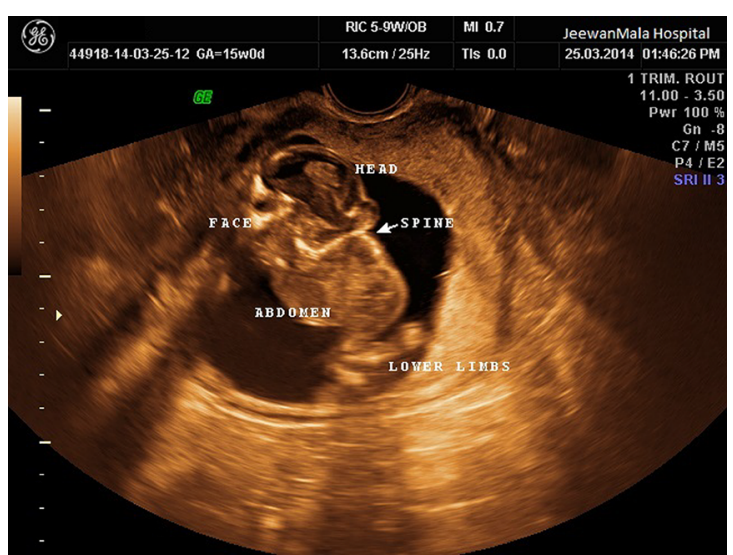

Fig. 1 Star gazing fetus so iniencephaly apertus

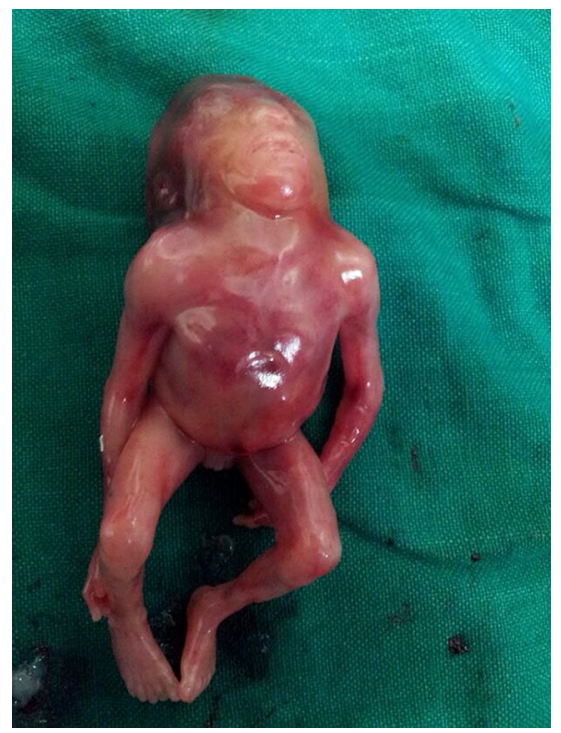

Fig. 2 Fetus after birth (supine) 
normal. Positive serum titres of $\operatorname{IgG}$ against toxoplasma and rubella were found. Ultrasonography (USG) was performed (GE Voluson using broad band 2-5 MHz convex transducer and $4-8 \mathrm{MHz}$ convex volume transducer). Grayscale scan revealed fetal intrauterine growth retardation. Neck was short and hyper-extended with fixed spinal retroflexion "star gazing" appearance. There was fusion of upper cervical vertebrae with the occiput, along with approximately $9.7 \mathrm{~mm}$ occipital encephalocele (Fig. 1). Spine was very short, and lower thoracic and lumbosacral vertebrae could not be visualized indicating complete rachischisis. Hands and feet appeared normal. However, the fetus did not demonstrate any limb activity during the examination period of 30 min with possible fetal akinesia. An antenatal diagnosis of inencephaly apertus was made. In view of obvious fetal anomalies incompatible with extra-uterine life, the parents were counseled for medical termination of pregnancy.

Examination of the abortus revealed a dead female fetus weighing $32 \mathrm{gm}$ (Fig. 2). Postnatal examinations confirmed antenatal USG findings. Radiograph (fetogram) showed an abnormally short spine with hemivertebrae and open spinal defect. The limb bones were normal. Karyotype analysis revealed normal chromosomal pattern.

Keywords Anencephaly, Iniencephaly, Apertus, Clausus, Neural tube defect

\section{Role of 3D Ultrasound in Imaging Uterine Anomalies: 3D Assessment of Cervix in Septate Uterus}

\section{Lakshmy Ravi Selvaraj}

Shri Lakshmi Clinic and Scan Centre, Krishnagiri, Tamil Nadu, India

\section{e-mail: drlakshmiravi@gmail.com}

Introduction Mullerian duct anomalies are known to cause a higher incidence of infertility, repeated firsttrimester abortions, fetal growth retardation, fetal malposition, preterm labor, and retained placenta. The role of imaging is to help detect, diagnose, and distinguish surgically correctable forms of mullerian duct anomalies from inoperable forms. Three dimensional transvaginal ultrasonography of the uterine cavity is extremely accurate in diagnosing and classifying anomalies. Hysterosalpingogram (HSG) and 2D ultrasound do not give a definitive diagnosis among bicornuate, septate, and uterus didelphys which has radically different measures of management.
Methods Different forms of Mullerian anomalies were examined by transvaginal 3D ultrasound. HSG and MRI both are gold standard in detecting uterine anomalies but 3D ultrasound proved a simple cost effective tool in detecting uterine anomalies.

In routine HSG, most often, septate uteri with double ostia are reported as unicornuate uterus as the dye passes through only one ostium. Cervical ostia can be assessed by $3 \mathrm{D}$ ultrasound and is illustrated in this paper. A collection of 11 cases of septate uterus with double ostium and their corresponding cervices are reviewed.

Results Of 2762 pelvic scans done over a period of three years, 87 cases of uterine anomalies were identified; $30 \%$ of these patients had come for evaluation of infertility; $10 \%$ had been incidentally detected, and the remaining $60 \%$ had come for evaluation of recurrent pregnancy loss. Seven patients with uterine anomalies had been detected at the time of early pregnancy scan.

With the help of 3D transvaginal scanning, the uterine anomalies were classified as follows: 15 arcuate uteri, 17 bicornuate uteri, 4 unicornuate uteri, and the remaining 44 were septate uteri. Out of the 17 bicornuate uteri, 6 were associated with rudimentary horn and 11 had equally well-developed horns. Assessment of cervix in 44 septate uteri revealed single ostium in 33 cases and double ostium in 11 cases.

Reabsorption anomaly of the uterus i.e., septate uterus was the highest, accounting for about $50 \%$ of the total uterine anomalies detected. Of 44 septate uteri, 33 had a uterine septum extending from the uterine fundus to varying lengths into the uterine cavity and had a single cervical ostium. Eleven were identified to have a fullthickness septum with double ostia. In the presence of full-thickness septum extending up to the cervix, the cervical canal was split into two by the septum.

Two of the septate uteri with double ostia were reported as unicornuate uterus on HSG as the dye injected had passed through only one ostium. Both of these patients had been evaluated for recurrent pregnancy loss and had three successive abortions. Ultrasound evaluation revealed a bifid endometrial cavity and 3D ultrasound clearly depicted that it was a septate uterus and not unicornuate uterus.

Conclusion The treatment options for septate uterus are very simple which is just a hysteroscopic resection of septum and 3D ultrasound is very sensitive in helping to differentiate this group of patients. Assessment of cervical ostia can be done with 3D ultrasound and is very useful in preoperative planning and decision making. The thickness and length of the septum can be assessed and rendering of the cervix can be done to demonstrate cervical ostium.

Keywords 3D ultrasound, Uterine anomaly, Septate uterus, Cervical ostium, Mullerian duct anomaly 


\section{Imaging in Polyhydramnios}

\section{Kazi Amir Irfan ${ }^{1, \#, ~ Y a s m i n ~ I q b a l ~}{ }^{2}$}

${ }^{1}$ Department of Radiology, Deccan College of Medical Sciences, Hyderabad, India; ${ }^{1}$ Department of Radiology, The Muslim Maternity and Children's Hospital, Hyderabad, Telangana, India; ${ }^{2}$ Department of Obstetrics and Gynecology, The Muslim Maternity and Children's Hospital, Hyderabad, Telangana, India

\#e-mail: kaziamirirfan@gmail.com

Background Polyhydramnios is defined as excess of amniotic fluid, greater than 1500 to $2000 \mathrm{~mL}$ with amniotic fluid index greater than 24. Polyhydramnios in pregnancy is a cause of concern for the treating physician as well as the patient. A thorough evaluation of the mother and fetus may help in identifying a cause which helps in appropriate management. In a significant proportion of patients, in whom no cause is found, reassurance counseling regarding the complications can be given to patients, which goes a long way in allaying their fears and misconceptions.

Objective The aim of the study was to identify maternal polyhydramnios, and then evaluate for fetal, placental, and maternal causes.

Materials and Methods All pregnant women (1800) who presented to the ultrasound department for second and third trimester scans were studied from March 2013 to April 2014. The liquor was measured objectively, using the 'four-quadrant' technique.

Results The etiology of polyhydramnios was idiopathic (68\%), fetal causes $(22 \%)$, maternal causes $(10 \%)$ and placental causes $(0 \%)$. Eighty $(4.4 \%)$ women had polyhydramnios $(4.4 \%)$. Fetal causes were seen in 18 $(22.5 \%)$ cases. Six cases were involving the gastrointestinal system, with two fetuses showing cleft lip, three cases of congenital diaphragmatic hernia and one case of proximal jejunal atresia. There were five neurological causes, with three cases of Chiari II malformation with spina bifida, one case of corpus callosum agenesis and one case of holoprosencephaly. There were three cardiac anomalies, with one case of hypoplastic left heart syndrome, one case of tetralogy of Fallot and one complex cardiac anomaly with hydrops in one fetus. There were two cases of unilateral renal pelviureteric junction obstruction, one case of Turner syndrome and one case of monochorionic twins with twin-twin transfusion syndrome. Maternal diabetes was seen as a cause in eight $(10 \%)$ women, in which one mother had a baby with macrosomia. Rest of the women revealed no obvious cause. Among these women with idiopathic polyhydramnios, fetal macrosomia was seen in two fetuses.

Conclusion Polyhydramnios is a common condition in pregnancy. Often it is an isolated finding. However, it can also herald maternal, placental, and fetal abnormalities thus detailed ultrasound evaluation is recommended in every case.

Keywords Polyhydramnios, Fetal anomalies, Diabetes mellitus, Macrosomia, Idiopathic polyhydramnios

\section{First Trimester Evaluation of Fourth Cerebral Ventricle}

\section{Lakshmy Ravi Selvaraj}

Shri Lakshmi Clinic and Scan Centre, Krishnagiri, Tamil Nadu, India

e-mail: drlakshmiravi@gmail.com

Introduction The fourth cerebral ventricle which is the intracranial translucency can be easily seen in the midsagittal plane of face used to measure the nuchal translucency. Obliteration of intracranial translucency has been documented as a marker in the early diagnosis of spina bifida.

The fourth ventricle is the window to detect posterior fossa malformations. It can be obliterated when there is an Arnold-Chiari malformation associated with open spina bifida or it could be enlarged when there is a posterior fossa anomaly-Dandy-Walker spectrum. The fourth ventricle looks prominent when there is cerebellar hypoplasia. This paper illustrates how evaluation of intracranial translucency had been useful not only in diagnosis of early spina bifida but also in early diagnosis of posterior fossa anomaly.

Methods The authors report a prospective analysis of 19 cases which had been detected with abnormal appearance of fourth cerebral ventricle in 12-14 week fetuses during routine aneuploidy screening. Acquisition of three dimensional volume by transvaginal sonography had been very useful in evaluating the fourth cerebral ventricle abnormality in these cases. Multiplanar imaging of the fourth ventricle had been of diagnostic importance and images of fourth ventricle in all three planes have been illustrated in all these abnormal cases. Results In eight cases, the absence of the intracranial translucency provoked the examiner to undertake a detailed evaluation of fetal spine (Fig. 3). An open spinal defect was detected as early as 12 weeks in all of these cases (Fig. 4).

In three cases of obliterated intracranial translucency, there was spectrum of other malformations associated with spinal defect. One was cloacal anomaly, the other was omphalocele-exstrophy-imperforate anus-spinal defects (OEIS) complex and the third was mid-line disruption sequence. 


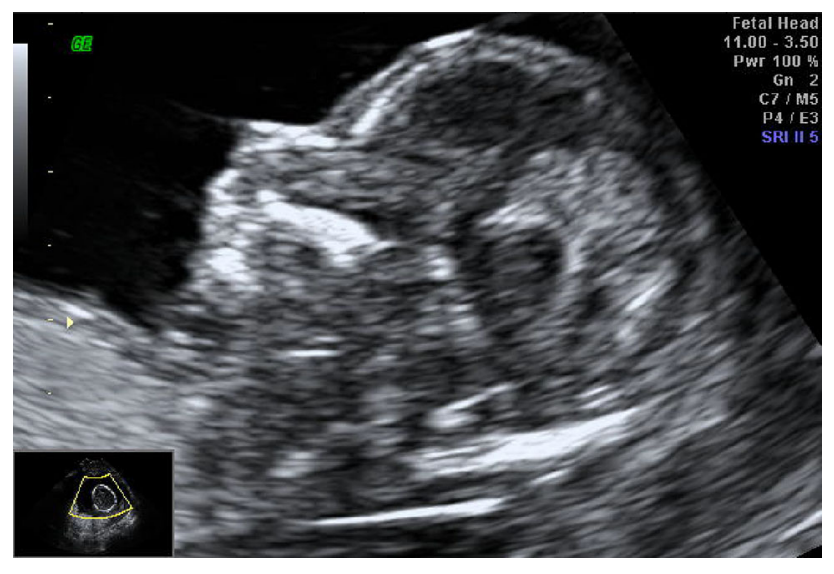

Fig. 3 Obliteration of intracranial translucency

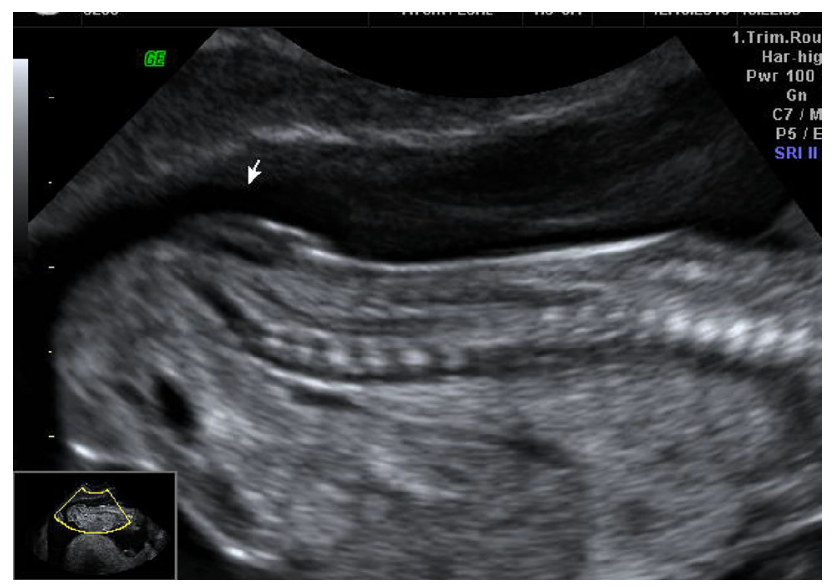

Fig. 4 Open spina bifida

In one case of abnormal intracranial translucency, the spine looked normal at 12 weeks. However, a follow-up scan at 18 weeks showed the cystic meningomyelocele.

In three cases, the intracranial translucency had been obliterated by a posterior fossa cyst suggesting posterior fossa anomaly. Evaluation of intracranium revealed ventriculomegaly in all three cases. One of these had multiple pterygium syndrome and the other two had karyotypic abnormality associated with cleft-lip and palate. One case which had an enlarged fourth ventricle had Chiari malformation and a small occipital encephalocele detected at 12 weeks.

The fourth ventricle looks prominent when there is cerebellar hypoplasia. Three cases with an abnormally enlarged intracranial translucency, showed evidence of cerebellar hypoplasia. Two of them had neuroarthrogryposis, one had the recurrence of this disorder in previous pregnancy and the diagnosis of the recurrence could be made as early as 12 weeks. Cerebellar hypoplasia was reconfirmed in the other case at 20 weeks. One of them with an enlarged intracranial translucency presented with retrognathia and bilateral cleft-lip and -palate.

Conclusion The inclusion of intracranial translucency during first trimester aneuploidy screening aids not only in the early diagnosis of spina bifida but also in the earlier detection of posterior fossa anomaly. An enlarged intracranial translucency should provoke the examiner to look for other signs of trisomy 13 and 18.

Keywords Fourth ventricle, Intracranial translucency, Posterior fossa, Spina bifida

\section{Interstitial Laser for Selective Fetal Reduction (sFR) in Type II Selective Fetal Growth Restriction in Monochorionic Twin Pregnancy}

\section{Vatsla Dadhwal $^{\#}$, Aparna Sharma, Dipika Deka}

Depatment of Obstetrics \& Gynecology, All India Institute of Medical Sciences, New Delhi, India

\#e-mail: vatslad@hotmail.com

Background sFGR is an important complication of MC pregnancy, with significant risk of intrauterine death (IUD) or neurological adverse outcome for both twins. Type II is characterized by persistent absent/reversed diastolic flow in umbilical artery. In a series of 27 cases, expectant management had IUD in $29.6 \%$ of intrauterine growth restriction (IUGR) cases and $22.2 \%$ larger twins. Neurological damage occurred in $14.8 \%$ and mean gestation at delivery was 28 weeks.

Objective The authors present a case of sFR in type II selective fetal growth restriction (sFGR) in monochorionic (MC) twin pregnancy.

Methods A G2P1 was referred at 21 weeks of gestation with MC twins with type II sFGR and oligoamnios in IUGR fetus (Fig. 5). After counseling, the IUGR fetus was reduced by interstitial laser carried out with $400 \mathrm{~nm}$ diode laser under USG guidance targeting intrafetal portion of umbilical cord. Patient continued pregnancy to term, without complications and delivered a $2.4 \mathrm{~kg}$ healthy baby at 39 weeks (Fig. 6).

Conclusions sFR in complicated MC pregnancy requires vaso-occlusive techniques, interstitial laser being one of the alternatives.

Keywords Monochorionic twins, Reduction, Interstitial laser, Selective fetal growth restriction 


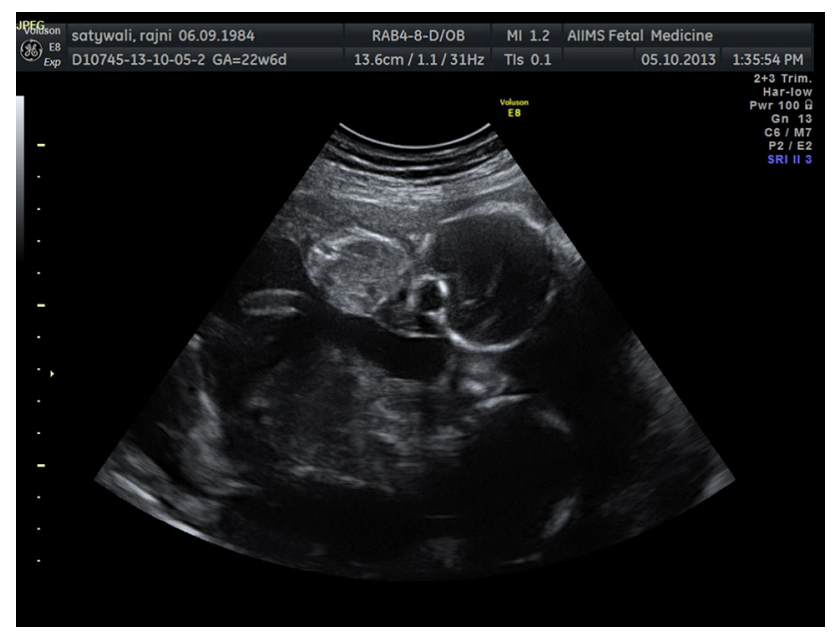

Fig. 5 MCDA twins with IUGR and anhydramnios in one twin

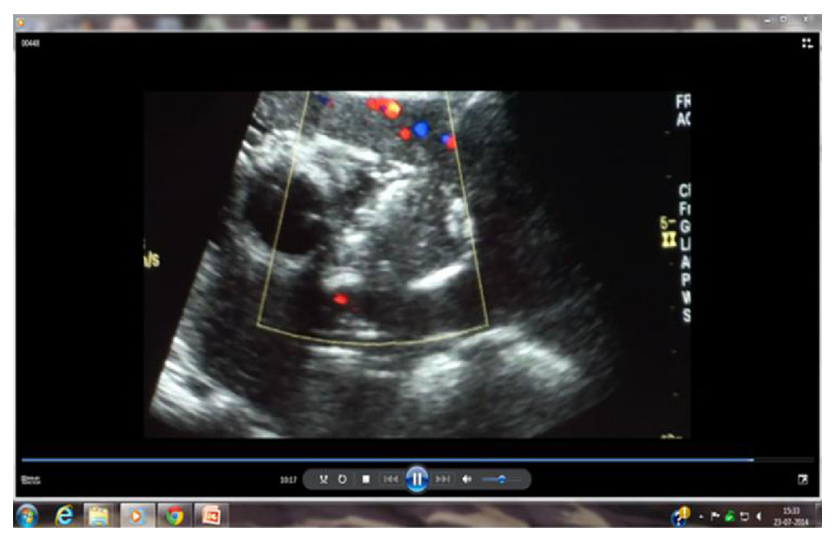

Fig. 6 Absent blood flow in reduced twin

\section{Velamentous Cord Insertion with Vasa Previa}

\section{Kazi Amir Irfan}

Department of Radiology, The Muslim Maternity and Children's Hospital, Hyderabad, India; Department of Radiology, Deccan College of Medical Sciences, Hyderabad, Telangana, India

e-mail: kaziamirirfan@gmail.com

Background Velamentous cord insertion is believed to result from the tendency of trophotropism in the placenta. It is seen in $1 \%$ of deliveries. Type I vasa previa is seen when there is velamentous cord insertion into placenta previa.

Objectives To present the ultrasound findings in a pregnant lady with velamentous cord insertion and vasa previa.
Report of Case A 25-year-old gravida 2, with a live child (born out of spontaneous vaginal delivery), presented for routine antenatal scan at 26 weeks gestational age. The fetal growth and morphology was normal. The liquor was adequate. Anterior placenta previa was seen with the lower end of placenta about $1.0 \mathrm{~cm}$ from the internal os. The cord showed a membranous insertion with fetal vessels traversing through the membranes along the margin of the internal os and entering the placenta. These findings were suggestive of velamentous cord insertion with vasa previa. The patient was informed about the diagnosis and counseled in detail regarding the complications. She was advised hospital admission at 30 weeks. When she came for routine antenatal checkup at 30 weeks, the lady refused admission because of financial reasons. She came to the hospital in the 34th week of pregnancy with bleeding per vaginum. As the cause of bleeding was already known, the lady was taken up for emergency cesarean section. The ultrasound findings were confirmed on surgery. Unfortunately, the fetus could not be saved. The maternal outcome was uneventful.

Discussion In velamentous cord insertion, the umbilical cord inserts into membranes, often adjacent to the placenta. It results due to trophotropism in which, parts of placenta grow while other parts atrophy. It can be seen on grayscale but is much better demonstrated on Doppler examination. Monochorionic twins are at an increased risk of developing velamentous cord insertion.

Vasa previa occurs when fetal vessels cross the internal os of the cervix underneath the fetal membranes. It can occur secondary to a succenturiate lobe where fetal vessels may traverse along the internal os when passing from the accessory lobe to the main placenta. It may also result secondary to velamentous cord insertion in a case of placenta previa. It is much better depicted on transvaginal scan with Doppler.

If velamentous cord insertion is not diagnosed antenatally, there is $60-80 \%$ chance of fetal mortality as the rupture of fetal vessels leads to fetal demise. Therefore, cesarean section is advised in such cases.

Conclusion Velamentous cord insertion with vasa previa is a rare condition, which when missed antenatally has a high fetal mortality rate. Therefore, this condition should be carefully looked for, especially in the settings of lowlying placenta. The fetus should be delivered only through cesarean section electively before the onset of labor.

Keywords Vasa previa, Velamentous cord insertion, Antenatal diagnosis, Antepartum hemorrhage, Fetal mortality 


\section{Ultrasonography in Detection of Congenital Anomalies in a Rural Population}

\author{
Aliva A. Nayak ${ }^{\#}$, Shivali Kashikar \\ Department of Radio-Diagnosis, Acharya Vinobha \\ Bhave Rural Hospital, Datta Meghe Institute of Medical \\ Sciences, Sawangi (Meghe) Wardha, Maharashtra, India \\ \#e-mail: alivanayak@rediffmail.com
}

Background Congenital malformations represent defect in morphogenesis during early fetal life. According to the World Health Organization (WHO) document of 1972, the term congenital malformations should be confined to structural defects at birth. In India where the social support system is virtually poor, bringing up a child with mental or physical handicap is a major burden on the parents and family. Ultrasonography is an ideal and cost-effective investigation in detecting congenital anomalies at an early stage. Congenital fetal anomalies are dreadful things for parents to know about, but an early detection may help to identify the severity of the disease, its outcome, and in certain cases, future planning to avoid birth of deformed babies.

Objectives To detect spectrum and incidence of congenital anomalies with the help of ultrasonography in a rural population.

Methods The study included 2280 women attending the antenatal clinic who were referred for routine ultrasound screening. The equipment used was Siemens Acuson 300 brilliance 190p. Routine obstetrical examination in terms of biparietal diameter, head and abdominal circumferences, and femur length along with amniotic fluid index, placental location and grading, and expected fetal weight were examined.

Results Congenital anomalies were detected in 57 (2.5\%) patients out of 2280 pregnant females. The anomalies were categorized into central nervous system, cardiovascular system, genito-urinary system, gastrointestinal system, skeletal system, respiratory system, syndromes, and miscellaneous anomalies. Maximum number of congenital anomalous cases detected was of genito-urinary system which was seen in 18 patients with multicystic dysplastic kidney being the most common, seen in seven cases followed by three cases of pelvi-ureteric junction obstruction, two cases each of posterior urethral valve and pelvic kidney and one case each of unilateral renal agenesis, hydroureter, ureterocele, and undescended testes. The second commonest system to have congenital anomalies was central nervous system, which was seen in 15 patients followed by seven cases of gastro-intestinal system, two cases each of skeletal and cardiovascular systems, one case of respiratory system, four cases of syndromes including one Turner, one Meckel-Gruber and two twin-twin transfusion syndromes, eight miscellaneous cases including two cases each of cleft-lip, umbilical varix, and hydrops fetalis and one case each of umbilical cord cyst and increased nuchal translucency.

Conclusion Congenital anomalies involving various systems were detected. The incidence of congenital anomalies in the pregnant females studied was $2.5 \%$, with maximum number of congenital anomalies seen in the genito-urinary system followed by central nervous system. Thus, an efficient ultrasound examination can detect fetal abnormalities early in the pregnancy so that the termination of pregnancy is safer with diminished economical and emotional costs, both for the medical system and the couple. Secondly, detailed exploration of the affected fetuses and diagnosis of all malformations can have an important role in the prenatal advice of future pregnancies as well. Hence, ultrasonography has an important role to play in the course of pregnancy by making the period of pregnancy safer for mother and child.

Keywords Biparietal diameter, Amniotic fluid index, Central nervous system, Cardiovascular system, Pelviureteric junction

\section{Agenesis of Ductus Venosus: A Review of 11 Cases}

\section{Lakshmy Ravi Selvaraj}

Shri Lakshmi Clinic and Scan Centre, Kaveripattinam, Krishnagiri, Tamil Nadu, India

e-mail: drlakshmiravi@gmail.com

Introduction Absence of ductus venosus (DV) is considered as a rare anomaly. DV is a conduit for the oxygenated blood to reach the heart. Major portion of umbilical venous flow is shunted through the DV into the heart and remaining through the portal vein into the liver. Routes of umbilical venous return in agenesis of DV could be extrahepatic or intrahepatic. In both patterns, the preferential direction of the flow towards the foramen ovale is not present.

The umbilical vein drains exclusively into the left branch of the intrahepatic portal vein in intrahepatic drainage with absence of ductus. Extrahepatic venous drainage is bypassing the liver where the umbilical vein directly connects to the iliac vein, the inferior vena cava (IVC), the renal vein, the right atrium or the coronary sinus. Extrahepatic venous drainage may be associated with congenital absence of portal venous system and volume overload of the heart with signs of cardiac failure and development of hydrops. Though there is less hemodynamic compromise in intrahepatic drainage, 
agenesis of ductus is commonly associated with congenital cardiac anomalies, chromosomal anomalies (Turner) and syndromic anomalies (Noonan). Prognosis depends on the presence of associated anomalies.

Materials and Methods This paper reviews 11 cases of agenesis of DV-four of the extrahepatic type and seven of the intrahepatic type. Four of the intrahepatic type were diagnosed in the first trimester.

Results Among the four extrahepatic cases, one had absent DV in which the umbilical vein drains into the internal iliac vein associated with complete absence of portal venous system and minimal ascites (Fig. 7). In this form of extrahepatic venous drainage, there is reduced perfusion and oxygenation of the liver parenchyma predisposing to agenesis of portal system and there is volume overload of the heart predisposing to high output cardiac failure and hydrops. In two cases, the umbilical vein directly drained into IVC, in the fourth case the umbilical vein ascended upwards and drained into the superior vena cava (SVC). This case was associated with major cardiac malformation.The other two were isolated and had normal outcome.

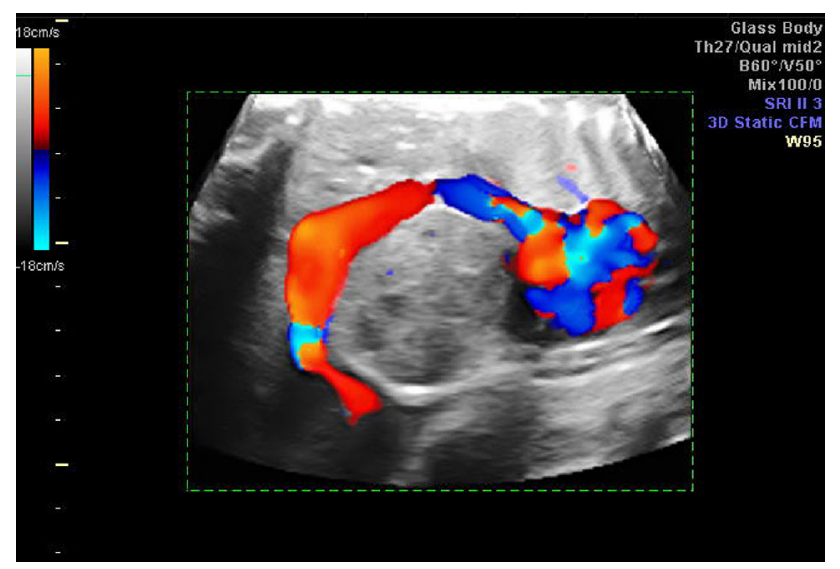

Fig. 7 Extrahepatic drainage of umbilical vein into inferior vena cava

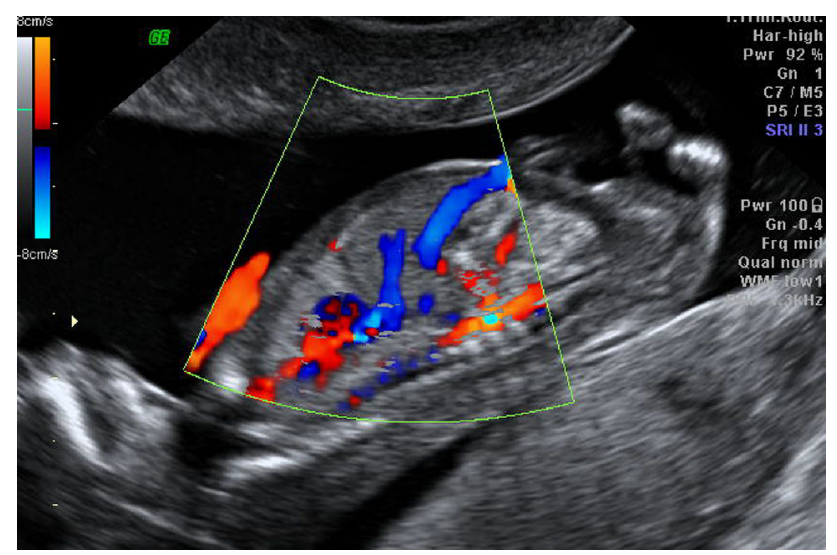

Fig. 8 Intrahepatic drainage of umbilical vein
Of the three cases of the intrahepatic type, one was associated with Fallot's tetrology while two others had a normal outcome (Fig. 8). Of the four cases diagnosed in the first trimester, one was hydropic and karyotyping revealed Turner syndrome. Two of them had major cardiac defects, and one had a normal outcome.

Conclusion Careful assessment of the DV and the umbilical vein should be a part of evaluation of every fetus with unexplained cardiomegaly and hydrops. All fetuses with abnormal connection of the umbilical vein should undergo assessment both in utero and after birth to exclude any cardiac or extracardiac abnormalities.

Keywords Ductus venosus, Agenesis, Umbilical vein, Hydrops

\section{Early Diagnosis of Interstitial Pregnancy at 6 Weeks}

\section{Chander Mohini $^{\text {1\# }}$, Sarita Aggarwal ${ }^{2}$, Tanya Aggarwal $^{2}$}

${ }^{1}$ Department of Radiodiagnosis, MM Institute of Medical Sciences \& Research, Mullana, Distt. Ambala, Haryana, India; ${ }^{2}$ Department of Obstetrics and Gynaecology, MM Institute of Medical Sciences \& Research, Mullana, Distt. Ambala, Haryana, India \#e-mail: chandermohini15@gmail.com

Background Interstitial pregnancy accounts for 2-4\% of all tubal pregnancies and has a mortality of $2 \%$. Sonography today is a vital diagnostic tool for the early diagnosis of interstitial pregnancy even before the clinical triad of signs and symptoms including abdominal pain, vaginal bleeding, and hemorrhagic shock setting in.

Objective Early diagnosis of interstitial pregnancy can be life saving as it has seven times higher mortality than those with ectopic gestations in general.

Report of Case A 36-year-old primigravida with amenorrhea of six weeks presented with vague heaviness in lower abdomen. Urine test for pregnancy was positive. The transvaginal sonography (done using 5-7.5 MHz) revealed uterine cavity empty. No gestational sac/mass seen in the extrauterine part of the fallopian tubes either. On meticulous sonographic survey of cornual regions of uterus, evidence of gestational sac corresponding to 4-5 weeks gestation, seen eccentrically located high in the fundus of uterus on right side, surrounded by myometrial mantle of less than $5 \mathrm{~mm}$ on all sides. This raised the possibility of interstitial ectopic pregnancy. On color Doppler flow imaging (CDFI), a ring of vascularity was seen around the sac.

Surgery showed an asymmetrical bulge in the right upper corner of uterus-suggestive of intact gestational 
sac of interstitial ectopic pregnancy, which was scooped out through an incision in the myometrium without resecting a portion of the tube.

Discussion and Conclusion Diagnosis of interstitial ectopic pregnancy is usually difficult and often delayed, resulting in high morbidity and mortality. In the present case, diagnosis of interstitial ectopic pregnancy was suggested on transvaginal sonography prior to the development of any complication resulting in early management with favorable outcome. This case thus emphasizes the role of ultrasonography in suspected ectopic pregnancy to diagnose the atypical site of ectopic pregnancy and safe and early management.

Keywords Interstitial pregnancy, Gestational sac, Transvaginal sonography, Color Doppler flow imaging (CDFI), Morbidity

\section{Fluorescence In Situ Hybridization in Failed Cultures of the Aborted Material can Increase the Diagnostic Yield of Aneuploidies}

\section{Anju Joshi", Preeti Paliwal, Meena Lall, Pushpa Saviour, Surbhi Mahajan, Nitika Sethia, Jyoti Jindal, Ishwar Chander Verma}

Center of Medical Genetics, Sir Ganga Ram Hospital, New Delhi, India

\#e-mail: anjujoshi009@gmail.com

Background Of all the clinically recognized pregnancies, 25-30 \% end in abortion. Up to 50-60\% of spontaneous first trimester miscarriages result from numerical chromosomal abnormalities. Chromosomal defects are found in 8-10\% of stillbirths, and $5 \%$ of couples experiencing recurrent pregnancy loss have an underlying chromosomal structural aberration, which can contribute to formation of abnormal gametes. Detection of chromosomal aberrations in the aborted material can provide a diagnosis and reduce the underlying cost by precluding further evaluation of other causes of the pregnancy loss.

Objective Evaluation of the frequencies of numerical and structural chromosomal abnormalities in aborted material is done by karyotyping, which remains a gold standard. But all tissues received in the laboratory were not in an appropriate condition to grow for subsequent karyotyping. Such samples were processed by fluorescence in situ hybridization (FISH) for detection of aneuploidies of chromosomes 13,16,18, 21, 22, X, and Y to ascertain a chromosomal cause of the abortion. This increased the diagnostic yield of chromosomal abnormalities and helped in counseling the couple for recurrence risk.
Methods Products of conception, skin, or chorionic villi were collected in a sterile transport media or normal saline. Cultures were set up for subsequent karyotyping, using ISCN 2011. In samples which failed to culture, FISH with chromosomes 13, 16, 18, 21, 22, X, and Y was done

Result Out of 500 samples, $276(55.2 \%)$ were successfully cultured and karyotyped and 224 (44.8\%) samples which failed to grow were subject to FISH.

Out of 276 karyotypes, 50 (18.11\%) had chromosomal abnormalities-42 (15.2\%) aneuploidies, 8 (2.89\%) structural chromosomal rearrangements. Remaining 226 (81\%) apparently had normal karyotypes.

Out of 224 samples subjected to FISH, 38 (16.96\%) had aneuploidies (Table 1).

Therefore, in total, 88 samples $(17.6 \%)$ showed chromosomal abnormalities (Table 1).

Conclusion FISH test in specimens with failed cultures increases the diagnostic yield of aneuploidies and helps in recurrence risk counseling for future pregnancy management. The whole genome array CGH may be a better option, for faster results without failed cultures problems but it is expensive. Therefore, karyotyping is still being used as the first test and we suggest that when the cultures fail, FISH test should be deployed.

Table 1 Abnormalities detected by culture and FISH $(\mathrm{n}=500)$

\begin{tabular}{lllll}
\hline & Culture & FISH & Total & $\begin{array}{l}\% \\
\text { Frequency }\end{array}$ \\
\hline \multicolumn{2}{l}{$\begin{array}{l}\text { Chromosomal constitution } \\
\text { Total samples }\end{array}$} & & & \\
224 (44.8 \%) & $\mathrm{N}=500$ & & & \\
46, XX or 46XY/ & 226 & 186 & 412 & \\
$\quad$ normal FISH & & & & \\
Chromosomal & 50 & 38 & 88 & $17.6 \%$ \\
$\quad$ abnormality & & & & $(88 / 500)$ \\
Numerical & $\mathrm{n}=42$ & $\mathrm{n}=38$ & $\mathrm{n}=80$ & \\
$\quad$ (Aneuploidy) & & & & \\
Common aneuploidies & & & & \\
Monosomy 16 & 0 & 3 & 3 & 0.6 \\
XO & 4 & 6 & 10 & 2 \\
Trisomy 13 & 2 & 2 & 4 & 0.8 \\
Trisomy 16 & 3 & 5 & 8 & 1.6 \\
Trisomy 18 & 5 & 4 & 9 & 1.8 \\
Trisomy 21 & 3 & 7 & 10 & 2 \\
Trisomy 22 & 1 & 2 & 3 & 0.6 \\
XXY & 0 & 1 & 1 & 0.2 \\
Triploidy & 8 & 8 & 16 & 3.2 \\
Tetraploidy & 1 & 0 & 1 & 0.2 \\
Total (n) & 27 & 38 & 65 & 13 \\
\hline & & & & \\
\hline
\end{tabular}


Keywords FISH, Karyotype, Abortus, Abortion, Aneuploidy, aCGH

\section{Spectrum of Anomalies Detected at the First Trimester Screening in a Rural Clinic}

\section{Lakshmy Ravi Selvaraj}

Shri Lakshmi Clinic and Scan Centre, Kaveripattinam, Krishnagiri, Tamil Nadu, India

e-mail: drlakshmiravi@gmail.com

Introduction Implementation of first trimester screening programme in the rural periphery is a great challenge. Awareness of the aneuploidy screening programme is very low among the practising rural physicians. A simple first trimester nuchal translucency (NT) scan to all antenatal patients in the rural sector is still a dream. As we are now moving towards earlier detection of anomalies in the first trimester, the objective of this study is to evaluate the fetus for structural defects at the time of NT scan. The 11-14 week scan in the periphery was meant only for high-risk pregnancy and recruiting patients for routine NT scan itself has been extremely difficult.

Methods This paper is a review of the spectrum of anomalies detected in singletons enrolled for NT scan at the author's center for four years. Of 1756 patients included in the study, 67 fetuses were found to have structural anomalies. The structural defects in these fetuses are described. Results

- Neural tube defects top the list. Eight of them had anencephaly, four had encephalocele, one had anencephaly with iniencephaly, two had craniorachischisis and seven had open spina bifida

- Twelve of the fetuses had hydrops, of which, four had cardiac malformation

- Seven of the fetuses had ventral wall defects, a part of mid-line disruption sequence-body stalk anomaly

- Seven had isolated cleft-lip and -palate-three bilateral and four unilateral

- Six had major cardiac defects

- Five had genitourinary malformation, two had hydronephrosis, two had cloacal anomaly and one had bladder outlet malformation

- Two had arthrogryposis and one had limb defects

- Six had syndromal associations-multiple pterygium syndrome (2), Holt-Oram syndrome, also called hearthand syndrome (1), Meckel-Gruber syndrome (1) (Fig. 9a, b), short rib polydactyly syndrome (1), GoldenharGorlin syndrome (1).

Conclusion Aneuploidy screening and NT scan have paved the way for earlier detection of anomalies, the importance of the 11-14 week scan is emphasized. Though the NT scan
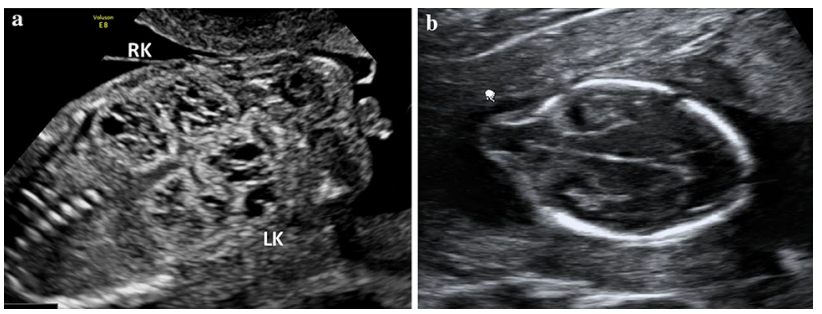

Fig. 9 Meckel-Gruber syndrome a Polycystic kidneys. b Occipital encephalocele

had been initially meant for aneuploidy screening it has now turned to be the decision making milestone in every pregnancy and should be made mandatory in every pregnancy.

Keywords First trimester, Anomaly, Syndrome, NTD, Aneuploidy screening

\section{First Trimester Biochemical Screening with Maternal Factors and Doppler Parameters to Predict the Risk of Pre- eclampsia in Indian Women}

Geetu Jhingan", Jyotsna Verma, Ratna D. Puri, Azad Singh, Divya Thomas, Sunita Bijarnia-Mahey, Ishwar Chander Verma

Center of Medical Genetics, Sir Ganga Ram Hospital, New Delhi, India

\#e-mail: Geetika_n81@hotmail.com

Objective Placental growth factor (PlGF), an angiogenic growth factor, has an impact on early placental vascular development. The main aim is to determine the levels of PIGF with PAPP-A in first trimester screening and study the effect of various maternal factors and Doppler findings on the risk of pre-eclampsia in Indian women. Method PlGF was measured in first trimester (gestation age: 11-14 weeks) with biochemical marker PAPP-A in about 500 pregnant women during the last two years. Markers were measured in serum on Delfia Xpress analyzer (Perkin Elmer) using fluroimmunoassay kits. Results were analyzed with respect to maternal age, body mass index, mean arterial blood pressure, hypertension, pre-eclampsia history and uterine artery Doppler parameters.

Results Out of 500 screening cases, 33 (6.6\%) were found to show high risk of pre-eclampsia. In our preliminary study, out of 33 screen positives, risk for late pre-eclampsia was seen in $90 \%$ cases. Only $10 \%$ cases showed risk for early pre-eclampsia. It was seen that increased body mass index, hypertension, urinary arterial pressure and previous history of pre-eclampsia are the main factors, which contribute significantly to 
low levels of PIGF. Low level of both PAPP-A and PIGF markers led to increased risk for pre-eclampsia. Conclusion In first trimester biochemical PAPPA screening, addition of early marker of placental failure i.e., PlGF has significant role in early prediction of patients who are at risk for pre-eclampsia and other placental dysfunction.

Keywords Pre-eclampsia, Biochemical screening, PlGF, PAPP-A, Doppler, Hypertension

\section{Prenatal and Postnatal Diagnosis of Lysosomal Storage Disorders}

\section{Jyotsna Verma, Sandeepika Sharma, Geetu Jhingan, Ratna D. Puri, Divya C. Thomas", Sunita Bijarnia- Mahay, Ishwar Chander Verma}

Center of Medical Genetics, Sir Ganga Ram Hospital, New Delhi, India

\#divcatthomas@gmail.com

Introduction Majority of the lysosomal storage disorders (LSDs) has no treatment and the cost of available treatments is out of reach for an average Indian family. To reduce the burden on the family and society, couples with previously affected child desire prenatal diagnosis in the next pregnancy.

Objective To offer the prenatal diagnosis to predict the affected and normal fetus in pregnant women with previous child affected with one of the LSDs at gestational age 11-14 weeks by enzyme assay in chorionic villi samples. Postnatal confirmation, if normal fetus is reported in previous prenatal result to reassure the parents. Method Prenatal diagnosis was done by enzyme assay in chorionic villi samples at gestational age of 11-14 weeks by fluorimetric method using artificial 4-methylumbelliferyl (4-MU) substrate and spectrophotometric method using 4-nitrocatechol substrates. Enzyme activity was reported in $\mathrm{nmol} / \mathrm{hr} / \mathrm{mg}$. Assays were done for the diagnosis of 23 LSDs (Gaucher, Fabry, Pompe, Niemann-Pick A/B, Tay-Sach, Sandoff, GM1, MPSI, II, IIIA, IIIB, IIIC, IIID, IVA, IVB, VI, VII, NCL 1, NCL2, Wolman, Fabry, Krabbe, MLD). Postnatal confirmation was done by enzyme assay in blood of the baby or telephonically.

Results The prenatal testing was done in 331 cases; 207 fetus $(67 \%)$ were normal and $124(37 \%)$ were affected. Postnatal enzymatic confirmation was done in 22 cases, all babies were found to be normal with one false negative for Krabbe disease due to the presence of $>25 \%$ residual activity. Further postnatal telephonic confirmation in $80 \%$ of the remaining cases revealed normal babies.
Conclusion Postnatal confirmation should be done to ensure the accuracy of the previous reported prenatal results and gain confidence in performing prenatal diagnosis for the complex lysosomal storage diseases.

Keywords Lysosomal storage disorders (LSDs), Lysosomal enzymes, Prenatal diagnosis (PND), Chorionic villi (CV), Postnatal confirmation

\section{First Trimester Fetal Echocardiography: Feasibility in Routine Practice}

\section{Lakshmy Ravi Selvaraj}

Shri Lakshmi Clinic and Scan Centre, Kaveripattinam, Krishnagiri, Tamil Nadu, India

e-mail: drlakshmiravi@gmail.com

Objectives Congenital cardiac malformations and chromosomal abnormalities are a leading cause of fetal malformations and pregnancy loss. Fetal echocardiography in the first trimester is a great challenge owing to the small size of the fetal heart. The basic four screening planes of the heart are difficult to obtain in all the fetuses at 11-14 weeks. However, the markers for aneuploidy done routinely in first trimester screening are nuchal translucency (NT), ductus venosus (DV), and tricuspid regurgitation (TR) also serve as markers for structural cardiac defects. Hence, the presence of an increased NT or an abnormal DV should provoke the examiner to take up a detailed examination of the fetal heart.

Methods The authors present a review of ten fetuses with cardiac anomalies detected in fetuses during routine aneuploidy screening. Five of them had an increased NT and three of them were hydropic. Volume rendering of the fetal heart with 4D spatio-temporal image correlation (STIC) had been extremely useful in confirmation of the anomaly thus improving diagnostic accuracy

Results The four chambered heart was the most useful diagnostic plane in detecting three cases of atrioventricular septal defect (AVSD) at 12 weeks. One of the fetuses had an unbalanced AVSD with small left ventricle. Large overriding aorta was the diagnostic clue in three cases of Fallot's tetrology. One of the fetuses showed flow reversal in pulmonary artery suggestive of pulmonary atresia. It also had bilateral radial ray defect suggestive of heart hand syndrome. Ventricular size discrepancy and abnormal three vessel trachea (3VT) view (single vessel) were the initial clues at 12 weeks for the detection of corrected transposition of the great arteries (cTGA) with pulmonary atresia confirmed at 16 weeks. Small left ventricle and flow reversal in the transverse aortic arch was found at 14 weeks in one case of hypoplastic left heart (Fig. 10a, b). Cardiomegaly 


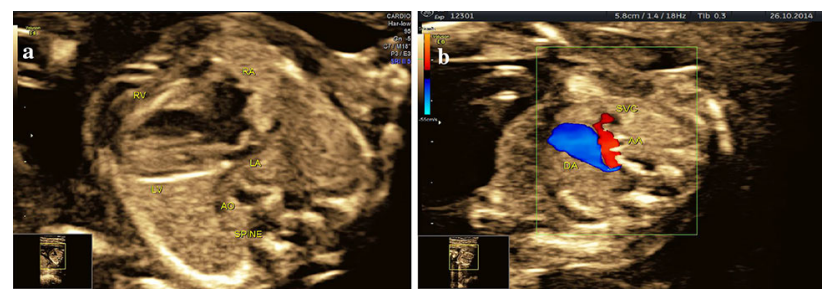

Fig. 10 Hypoplastic left heart a Small left ventricle in four chamber view. b Flow reversal in ascending aorta

with poor right ventricular filling and bi-directional flow in the aortic and pulmonary valves was detected at 12 weeks in one fetus with increased NT which went in for fetal demise the subsequent week. Subsequent karyotyping revealed trisomy 18 . Routine evaluation of the fetal heart also helped in picking up cardiac anomalies even in the absence of markers. Large ventricular septal defect (VSD) with hypoplastic left heart and double outlet ventricle could be diagnosed as early as at 12 weeks and was later confirmed at 16 weeks. Even in this case, the two diagnostic planes were the four chamber view and 3VT color. Four chambered heart showed the large VSD and the small left ventricle while the $3 \mathrm{VT}$ view showed a single vessel.

Conclusion First trimester evaluation of the fetal heart would be feasible, if attempted, and a four chamber and $3 \mathrm{VT}$ view could be imaged in almost all the fetuses presenting at the time of aneuploidy screening. The four chamber and $3 \mathrm{VT}$ view with color were the diagnostic clues in almost all these cardiac anomalies. Hence, the four chamber and 3VT view, if included in routine first trimester screening, would give the maximum yield in the detection of cardiac anomalies, especially, if there is an increased NT.

Keywords Fetal echo, Cardiac anomaly, Aneuploidy screening, First trimester screening

\section{Spectrum of Congenital Brain Anomalies}

\author{
Shivani Garg ", Shivali V. Kashikar \\ Department of Radiodiagnosis; Acharya Vinoba Bhave \\ Rural Hospital, Sawangi (Meghe), Wardha, \\ Maharashtra, India \\ \# email: shivani509garg@gmail.com
}

Background The incidence of congenital anomalies, out of 2000 patients scanned, was $4 \%$ and incidence of congenital brain anomalies was $1.9 \%$.

Objectives To determine spectrum and incidence of congenital brain anomalies

Methods Two thousand patients attending antenatal clinics were examined by ultrasound. Congenital brain anomalies were detected. Alive newborns were followed to confirm the diagnosis. Along with it, physical status of mother was also examined. In the present study, the equipment used was Siemens ACUSON 300 ultrasound system.

Results Two thousand patients were examined of which 80 had congenital anomalies, 38 had congenital brain anomalies, eight had anencephaly-acrania sequence, four had holoprosencephaly, four had ventriculomegaly, two had occipital encephalocele, two had microcephaly, two had schizencephaly, two had agenesis of corpus callosum, two had hypoplasia of corpus callosum, two had Chiari II malformation, two had porencephalic cyst, four had choroid plexus cyst, two had Dandy-Walker malformation, two had lissencephaly. Some of them had more than one anomaly.

Conclusion A variety of congenital brain anomalies were found. Occurrence of congenital brain anomalies was found to be $1.9 \%$ with maximum being anencephaly. Antenatal ultrasonography examination provided good results. Interruption of proper formation, growth, and migration of neurons and glial cells, early in development, can cause structural defects in brain and lead to brain malformation. It must be remembered that malformations can arise from a variety of causes and that not all of them are genetic. Destructive infections and ischemic in utero injuries may also result in abnormal formation of developing brain.

Keywords Ultrasound, Congenital, Brain anomalies, Antenatal, Anencephaly

\section{Prenatal Diagnosis of Isolated Median Cleft- Palate}

\section{Lakshmy Ravi Selvaraj}

Shri Lakshmi Clinic and Scan Centre, Kaveripattinam, Krishnagiri, Tamil Nadu, India

e-mail: drlakshmiravi@gmail.com

Introduction Orofacial clefting is one of the most common birth defect. Prenatal diagnosis of cleft-lip is easily obtained by conventional 2D sonography. Cleft-palate is more difficult to identify, especially, if it is an isolated anomaly. Ultrasound evaluation of the palate is problematic and due to the limitations of 2D sonography, the majority of palatine clefts are diagnosed in the neonatal period. Advantages of evaluating palate at 12 weeks is that there is less shadowing from facial bones and as the palate is flat and less concave at 12 weeks, it favors full visualization in multiplanar display. A perfect midsagittal view is mandatory for evaluating nuchal translucency (NT) and just a volume acquisition in this plane would take only a few more seconds. 
Methods 2D and 3D evaluation of face and palate were done for fetuses with crown rump length (CRL) greater than $50 \mathrm{~mm}$, as palatal fusion is completed by 12 weeks. The bony landmarks of the palate taken into consideration for screening palatine clefts were the appearance of the palate in mid-sagittal view, premaxillary triangle in the coronal view and the alveolar ridge of maxilla in axial view. After volume acquisition of the face in the mid-sagittal plane, rendering of the palate was done using the flipped face view (Fig. 11). Three dimensional volume imaging of the palate done combined with static volume contrast imaging to confirm the cleft. Omniview could also be used to image the palate. This methodology had been very useful in detecting 11 cases of cleftpalate associated with cleft-lip during first trimester aneuploidy screening in the past three years.

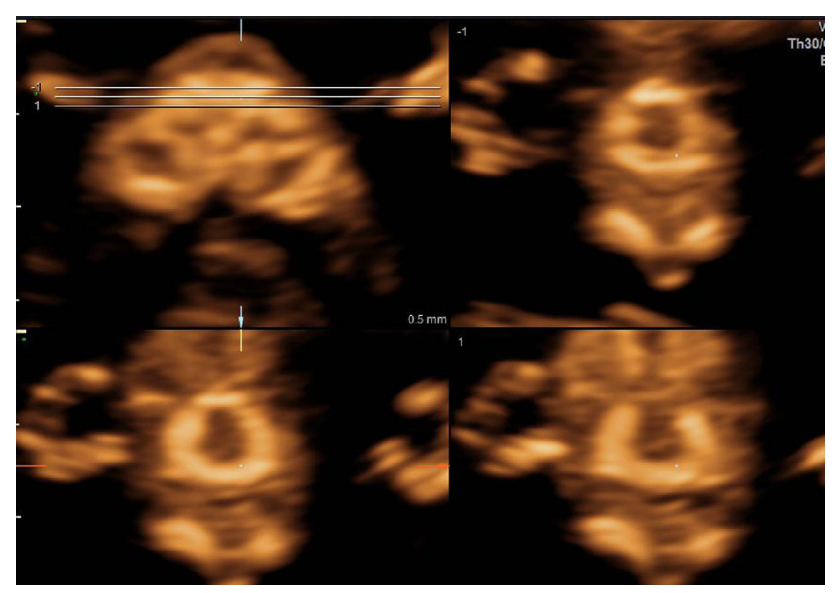

Fig. 11 Tomographic ultrasound image in coronal view shows intact palate in all the sections (normal palate)

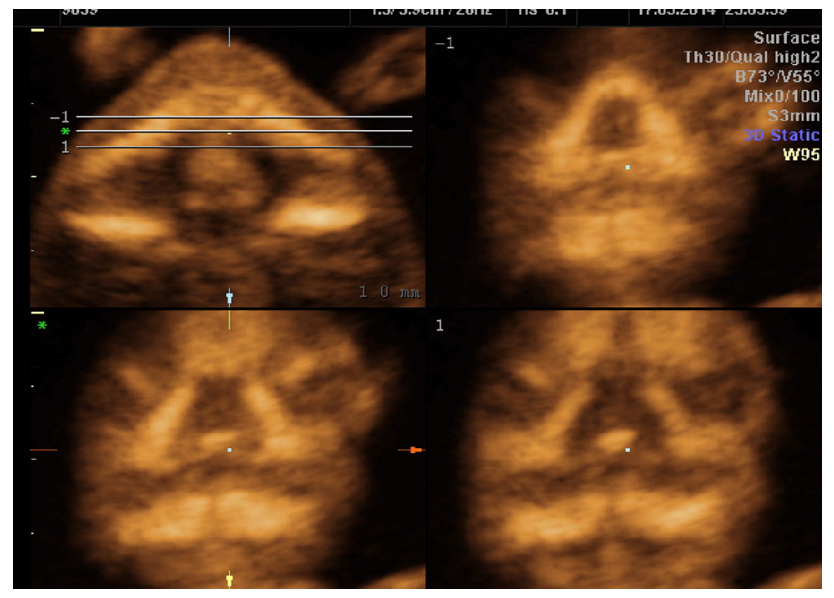

Fig. 12 Tomographic ultrasound image in coronal views shows intact premaxillary triangle but defect in the other sections (isolated median cleft palate)
Results Twenty-eight-year-old second gravid with a consanguinous marriage, reported for first trimester screening. The first child had severe retrognathia, cleft palate, high myopia and was provisionally diagnosed to have Stickler syndrome. The child was operated for cleft palate and had subnormal intelligence.

Ultrasound in the current pregnancy revealed a single live fetus of 13 weeks gestation. Isolated median cleft palate was detected at 13 weeks (Fig. 12). Evaluation of the face showed severe retrognathia. Volume acquisition done in the mid-sagittal plane and the bony landmarks of the palate were evaluated. Sagittal scroll of the acquired volume clearly revealed the defect in the secondary palate and the palatine defect was confirmed in multiplanar imaging. The patient was referred to a Fetal Medicine unit and Genetic clinic. They confirmed the findings and concurred with the diagnosis. After the counseling, the parents opted for termination. Karyotype of the fetus was done and was normal. DNA storage had been done for further genetic analysis. Autopsy revealed severe retrognathia and median cleft-palate was confirmed.

Conclusion The evaluation of the fetal palate is feasible and much easier when done at 12 to 14 weeks. Ultrasound diagnosis of the palate which is a very difficult task could be made easier when evaluation is shifted to the first trimester. Evaluation of the palate in the first trimester needs expertise and training but volume imaging and omniview have made this herculean task an easy one. Diagnosis of an isolated cleft-palate in future will no longer remain a mirage.

Keywords Median cleft-lip, Cleft-palate, Cleft-lip and palate, Ultrasound diagnosis, Isolated median cleft

\section{Invasive Prenatal Diagnostic Procedure in $\mathbf{5 8 6}$ Cases}

\section{Neeta Singh", Mandakini Pradhan}

Department of Maternal and Reproductive Health, Sanjay Gandhi Post Graduate Institute of Medial Sciences, Lucknow, India

\#e-mail: singhneeta76@gmail.com

Objective Antenatal procedures are effective prenatal diagnostic tools for detection of fetal disorders. The authors report their experience with antenatal procedures, focusing on the outcome of these procedures.

Materials and methods This is a retrospective study of pregnant women presenting at the Outpatient Department of Maternal and Reproductive Health, Sanjay Gandhi Post Graduate Institute of Medical Sciences, Lucknow between July 2009 and April 2014. The data 
were analyzed to find out the indications, gestational age, complications, and outcome of diagnostic prenatal testing.

Results Antenatal diagnostic procedures include amniocentesis, chorionic villous sampling (CVS), cordocentesis, vesicocentesis, and paracentesis. There were total 586 procedures done during this period, of which 79 were CVS (13.5\%), 362 were amniocentesis $(61.8 \%), 108$ were cordocentesis $(18.4 \%), 27$ were vesicocentesis $(4.6 \%)$, and 10 were paracentesis $(1.7 \%)$.

The indications for amniocentesis were 119 for raised risk for trisomy 21 in serum screening, 141 for abnormal genetic sonogram, 42 for abnormal genetic sonogram along with raised risk in serum screening, 39 for previous baby with chromosomal abnormality or family history of chromosomal or single gene disorder, 18 for parents with abnormal karyotype, and three for suspected fetal infections.

The indications for CVS included 18 for couples with both partners thalassemia trait, 15 for previous baby with thalassemia, 14 for abnormal ultrasonography, 10 for previous baby affected with muscle dystrophies, six for previous baby with hemophilia, seven for previous baby with sickle cell disease, three for suspected triploidy, leukocyte adhesion defect and congenital adrenal hyperplasia each.

The indications for cordocentesis were 72 for abnormal genetic sonogram, 12 for previous baby with chromosomal abnormality or family history of chromosomal or single gene disorder, 10 for previous baby with muscle dystrophy, suspected fetal anemia in six, five for previous baby with sickle cell disease and three for suspected partial molar pregnancy.

The indications for vesicocentesis included fetus with ultrasonography suggestive of urinary tract obstruction like posterior urethral valve in 14 , urorectal septal malformation in eight, hydroureteronephrosis in four and megacystis in one case. The indication for paracentesis was isolated fetal ascites in four patients.

Out of 586 patients, $381(65 \%)$ patients presented at gestational age less than or equal to 20 weeks and 205 patients presented after 20 weeks $(35 \%)$. Out of total procedures, $73(12.5 \%)$ had abnormal and 513 had normal results. Fifty seven patients had gestational age less than 20 weeks and all of them had termination of pregnancy.

Amongst all abnormal results, prenatal invasive diagnostic tests were able to diagnose 18 fetuses with trisomy 21, six with triploidy and translocation each, five fetuses with trisomy 18 and Turner syndrome each, four fetuses with trisomy 13 and three with Klinefelter syndrome.

With these tests, the authors detected six cases of fetal anemia (not due to isommunization), three fetuses with fetal cytomegalovirus (CMV) and two fetuses with rubella infection; five fetuses with thalassemia major, three with sickle cell disease and Duchenne muscle dystrophy each, two fetuses with hemophilia, and congenital adrenal hyperplasia.

All the patients with abnormal results had termination of pregnancy where gestational age was less than 20 weeks.

There was one case of preterm premature rupture of membranes with preterm delivery where amniocentesis was done at 32 weeks for a fetus which showed congenital diaphragmatic hernia with transposition of great vessels.

There were two cases of intrauterine death following cordocentesis. There were no complications following CVS and vesicocentesis.

Conclusion Prenatal invasive tests were effective in diagnosing and preventing birth of abnormal fetus.

Keywords Invasive prenatal testing, Amniocentesis, Chorionic villus sampling, Cordocentesis, Prenatal

\section{Prenatal Diagnosis of Down Syndrome}

\section{Vatsla Dadhwal $^{1}$, Dipika Deka ${ }^{1}$, Piyush Bansal ${ }^{1 \#,}$ Aparna Sharma ${ }^{1}$, Madhulika Kabra ${ }^{2}$, Neerja Gupta $^{2}$, Sumita Agarwal', Uruj Jehan', Kavita Khoiwal ${ }^{1}$}

${ }^{1}$ Department of Obstetrics and Gynecology;

${ }^{2}$ Department of Genetics; All India Institute of Medical Sciences, New Delhi, India

\#e-mail: drpiyushonline@gmail.com

Introduction Down syndrome is the most common aneuploidy. All women, regardless of age, are offered screening.The aim is to identify high-risk women and offer them diagnostic testing by chorionic villus sampling (CVS) [if less than 13 weeks of gestation] or amniocentesis [if beyond 15 weeks of gestation]. There is $0.5 \%-1 \%$ excess miscarriage risk for amniocentesis, and with CVS, it is $1 \%-2 \%$. If confirmed with Down syndrome, they may be offered a termination of pregnancy or they may continue with the affected pregnancy with support. The authors present observations in their unit at All India Institute of Medical Sciences (AIIMS) over the past five years.

Materials and Methods It is a retrospective study. Data were retrieved from register entries over the past five years at AIIMS in the authors' unit and genetics lab. The major indications for amniocentesis and CVS were searched.

Results The most common procedure performed was amniocentesis. In singleton population, 22 out of 476 cases were confirmed for Down syndrome on karyotype 
Table 2 Results of amniocentesis

\begin{tabular}{llrl}
\hline $\begin{array}{l}\text { S. } \\
\text { no. }\end{array}$ & Screening parameter & $\begin{array}{l}\text { No. of } \\
\text { cases }\end{array}$ & $\begin{array}{l}\text { No. of } \\
\text { Down } \\
\text { positive }\end{array}$ \\
\hline 1. & Advanced maternal age & 154 & 9 \\
2. & First trimester screen positive & 44 & 3 \\
3. & Second trimester screen positive & 185 & 8 \\
4. & One soft marker on USG \pm serum screen & 92 & 5 \\
5. & Two Soft Markers on USG \pm serum screen & 30 & 7 \\
6. & Previous baby with DS & 68 & 1 \\
\hline
\end{tabular}

$D S$ Down syndrome, USG ultrasonography

(4.6\% of screen positive patients). While in twin gestation, no case of Down syndrome was detected out of eight cases.

CVS as a method of diagnosis was less used (total number of procedures performed $=38$ ).

The results of amniocentesis are elaborated in Table 2.

Discussion Only $40 \%$ of patients positive for increased risk for Down syndrome are elderly gravidas (>35 years). Hence, screening should be offered to all age groups. The recurrence rate of Down syndrome after one baby with Down syndrome was found to be $1.4 \%$. The most common indication for amniocentesis has been the second trimester screen, as most patients present late. However, the first trimester integrated screen is a better option as it offers the advantage of early termination. Isolated single soft marker is not an effective screening strategy and at least two markers or an additional biochemical screen must be positive. Two soft markers on ultrasonography have a fairly good likelihood ratio of finding a baby with Down syndrome.

Keywords Down syndrome, Prenatal diagnosis, Amniocentesis, Chorionic villus sampling

\section{Maternal and Cord Blood Serum Insulin-like Growth Factor-1 (IGF-1) IGF Binding Protein-3 (IGFBP-3), in Asymmetrically Small-for- Gestational-Age Neonates}

\section{Amrit Gupta $^{1 \#}$, Nisha Singh², Swasti Tiwari ${ }^{3}$, Madan Mohan Godbole ${ }^{4}$}

${ }^{1}$ Department of Maternal and Reproductive Health, Sanjay Gandhi Postgraduate Institute of Medical Sciences, Lucknow, Uttar Pradesh, India; ${ }^{2}$ Department of Obstetrics and Gynecology, Chhatrapati Shahuji Maharaj Medical University, Lucknow, Uttar Pradesh, India; ${ }^{3}$ Department of Molecular Medicine, Sanjay Gandhi Postgraduate Institute of Medical Sciences,
Lucknow, Uttar Pradesh, India; ${ }^{4}$ Department of Endocrinology, Sanjay Gandhi Postgraduate Institute of Medical Sciences, Lucknow, Uttar Pradesh, India

\#e-mail: amrit.gupta14@gmail.com

Introduction The intrauterine fetal environment is crucial for fetal survival and long-term health. Placenta plays an important role in the exchange between mother and fetus and regulates the growth of the fetus via production and metabolism of growth-regulating hormones such as IGFs and glucocorticoids. This study was planned to investigate the relationship between birth weight and insulin-like growth factor-1 (IGF-1), IGF binding protein-3 (IGFBP-3), in neonates with normal growth (appropriate-for-gestational-age: AGA) and retarded growth (small-for-gestational-age: SGA).

Objectives To find the association of maternal and cord blood insulin growth factors 1 and 2, and IGFBP-3 in normal pregnancy and in pregnancy with intrauterine growth restriction (IUGR) of fetus.

Method This was a prospective case control study conducted in collaboration with a postgraduate instituteSanjay Gandhi Post Graduate Institute of Medical Sciences (SGPGIMS), Lucknow and a tertiary level hospital-King George's Medical University (KGMU), Lucknow, over a period of two years.

Inclusion criteria The control group included normal pregnant women with singleton pregnancy and growth parameters appropriate for gestational age. In the study group, all cases were diagnosed to have IUGR by the following criteria:

- Gestational age confirmed by ultrasonography in the first or early second trimester

- IUGR suspected by a lag of $>3$ weeks in fetal biometry on serial USG after 20 weeks

- Birth weight below 10th centile for the gestational age

- IUGR was confirmed after birth by weight in accordance with gestational age

Exclusion criteria Pregnant women having any of the following characteristics were excluded from the study

- Previous history of maternal complications, severe anemia, hematological diseases or diabetes

- Evidence of ultrasound markers for fetal chromosomal defects, or congenital anomalies

- Evidence of intrauterine infections such as fever, IgM positivity for TORCH infections, vaginal discharge

A total of 180 mothers were recruited; 100 serum samples were maternal and collected between gestational age of 32 and 38 weeks; 80 were cord blood from SGA and AGA fetuses collected soon after delivery. Sixty eight maternal samples and 51 cord blood samples were 
analyzed. IGF-I, IGFBP-3 were measured in maternal serum and venous cord blood at birth by chemiluminescent immunometric assay.

Results In maternal serum, the mean serum IGF-I levels was $251 \mathrm{ng} / \mathrm{mL}$ in the control group versus $214.48 \mathrm{ng} / \mathrm{mL}$ in the study group. The IGF-I levels were positively correlated with the birth weight. On the other hand, IGBP-3 was negatively correlated $(\mathrm{p}=0.0051 ; \mathrm{MS}=8.499)$.

In contrast, in cord blood, the serum levels of both IGF-I and IGFBP-3 were positively correlated to birth weight ( $\mathrm{p}<0.001$ and 0.1021 , respectively).
Conclusion Cord blood IGF-I and IGFBP-3 play an important role in the regulation of fetal and neonatal growth. It is likely that IGFBP-3 in maternal blood and IGF-1 and IGFBP-3 play an important role in the growth potential of the fetus.

Keywords Insulin growth factor-1, IGFBP-3, Maternal, Cord blood 